\title{
Renal Function and Death in Older Women: Which eGFR Formula Should We Use?
}

\author{
Muna T. Canales, ${ }^{1}$ Terri Blackwell, ${ }^{2}$ Areef Ishani, ${ }^{3}$ Brent C. Taylor, ${ }^{4}$ Allyson Hart, ${ }^{5}$ \\ Rebecca J. Beyth, ${ }^{6}$ and Kristine E. Ensrud ${ }^{4}$ \\ ${ }^{1}$ Malcom-Randall VAMC, Department of Medicine, University of Florida, Gainesville, FL, USA \\ ${ }^{2}$ Research Institute, California Pacific Medical Center, San Francisco, CA, USA \\ ${ }^{3}$ Department of Medicine, University of Minnesota, Minneapolis VA Health Care System, Minneapolis, MN, USA \\ ${ }^{4}$ Center for Chronic Disease Outcomes Research, Minneapolis VA Health Care System, Department of Medicine, \\ University of Minnesota, Minneapolis, MN, USA \\ ${ }^{5}$ Hennepin County Medical Center, Minneapolis, MN, USA \\ ${ }^{6}$ Malcom-Randall VAMC GRECC, Department of Medicine, University of Florida, Gainesville, FL, USA
}

Correspondence should be addressed to Muna T. Canales; muna.canales@medicine.ufl.edu

Received 18 October 2016; Revised 16 February 2017; Accepted 1 March 2017; Published 29 March 2017

Academic Editor: Ziyad Al-Aly

Copyright (C) 2017 Muna T. Canales et al. This is an open access article distributed under the Creative Commons Attribution License, which permits unrestricted use, distribution, and reproduction in any medium, provided the original work is properly cited.

\begin{abstract}
Background. The Berlin Initiative Study (BIS) eGFR equations were developed specifically for aged populations, but their predictive validity compared to standard formulae is unknown in older women. Methods. In a prospective study of 1289 community-dwelling older women (mean age 79.5 years), we compared the performance of the BIS1 SCr-based equation to the CKD-EPI cr $_{\text {and the }}$ BIS2 SCr- and Scysc-based equation to the CKD-EPI cr,cysc $_{\text {to }}$ tredict cardiovascular and all-cause mortality. Results. Prevalence of specific eGFR category (i.e., $\geq 75,60-74,45-59$, and $<45$ ) according to eGFR equation was $12.3 \%, 38.4 \%, 37.3 \%$, and $12.0 \%$ for BIS1; $48.3 \%, 27.8 \%, 16.2 \%$, and $7.8 \%$ for CKD-EPI ${ }_{\text {cr }}$, $14.1 \%, 38.6 \%, 37.6 \%$, and $9.6 \%$ for BIS2; and 33.5\%, 33.4\%, $22.0 \%$, and $11.1 \%$ for CKD$\mathrm{EPI}_{\text {cr,cysc }}$, respectively. Over $9 \pm 4$ years, 667 (51.8\%) women died. For each equation, women with eGFR $<45$ were at increased risk of mortality compared to eGFR $\geq 75$ [adjusted HR (95\% CI): BIS1, 1.5 (1.1-2.0); CKD-EPI ${ }_{c r}, 1.7$ (1.3-2.2); BIS2, 2.0 (1.4-2.8); CKD$\mathrm{EPI}_{\mathrm{cr}, \mathrm{cysc}}, 1.8(1.4-2.3)$; $p$-trend $\left.<0.01\right]$. Net reclassification analyses found no material difference in discriminant ability between the BIS and CKD-EPI equations. Results were similar for cardiovascular death. Conclusions. Compared to CKD-EPI, BIS equations identified a greater proportion of older women as having CKD but performed similarly to predict mortality risk. Thus, the BIS equations should not replace CKD-EPI equations to predict risk of death in older women.
\end{abstract}

\section{Introduction}

Reduced renal function defined by varying cut-points of estimated glomerular filtration rate (eGFR) in older adults has been associated with greater risk of cardiovascular (CVD) and all-cause mortality [1-3]. However, there is debate regarding the threshold of eGFR beyond which risk of death rises $[4,5]$. Much of this controversy is related to the insensitivity of serum creatinine (SCr) based estimates in older adults and the lack of eGFR formulae developed specifically for use in the aged population [6].

In an effort to move toward more accurate estimation of GFR across populations and age groups, the 2012 Kidney
Disease Improving Global Outcomes (KDIGO) guidelines called for a paradigm shift from use of the clinical standard Modification of Diet in Renal Disease (MDRD) formula to the Chronic Kidney Disease Epidemiology Collaboration creatinine $\left(\mathrm{CKD}-\mathrm{EPI}_{\mathrm{cr}}\right) 2009$ equation to estimate GFR in adults [7]. Furthermore, for the aged population in which SCr-based estimates may not be as sensitive, the guidelines recommend use of serum cystatin C (Scysc) based equations (CKD-EPI ${ }_{\text {cysc }}$ and CKD-EPI $I_{c r, c y s c}$ ) [7]. The CKD-EPI formulae were developed in a large population $(n=8254$ and 5352 for the CKD-EPI ${ }_{c r}$ and the CKD-EPI cystatin C equations, resp.) that pooled participants from various research studies and clinical cohorts. While the CKD-EPI cohorts reflected 
gender and racial/ethnic diversity (56-58\% male, $30-40 \%$ African-American) and a range of renal function (mean measured GFR $\left.68( \pm 40) \mathrm{mL} / \mathrm{min} / 1.73 \mathrm{~m}^{2}\right)$, they underrepresented older adults (mean age was $47 \pm 15$ years) $[6,8]$. Thus, KDIGO guidelines further recommended the use of alternate formulae that may perform better for certain populations with respect to estimation of GFR [7].

To that end, the Berlin Initiative Study 1 (BIS1, SCr-based) and BIS2 (SCr and Scysc-based) were the first eGFR formulae to be developed and internally validated in comparison to direct measures of GFR specifically in a population of older adults using a cohort of 570 community-dwelling older Caucasian German men and women over 70 years of age with mean measured GFR (range) $60(16-117) \mathrm{mL} / \mathrm{min} / 1.73 \mathrm{~m}^{2}$ [9]. These formulae represent the first attempt to tailor a formula to the aged population while also incorporating a more sensitive measure of renal function in the elderly, Scysc [9]. To date, however, no study has validated the performance of these equations against the KDIGO-recommended CKDEPI equations to predict the key outcome of death in older women.

Therefore, we studied 1289 participants enrolled in a multicenter, prospective study of disease outcomes in community-dwelling older women, the Study of Osteoporotic Fractures (SOF), who had existing measures of both SCr and Scysc, and compared the ability of the new BIS1 (SCr-based) and BIS2 (SCr and Scysc-based) equations to guidelinerecommended CKD-EPI ${ }_{c r}$ and CKD-EPI ${ }_{\text {cr,cysc }}$ equations, respectively, to predict incident all-cause and CVD death.

\section{Methods}

2.1. Participants. The SOF is a NIH-funded prospective cohort study of older women initially designed to determine risk factors for fractures and falls but expanded to evaluate risk for other outcomes including all-cause and cause-specific mortality in older women. SOF enrolled 9704 women aged $\geq 65$ years between September 1986 and October 1988 for participation in the baseline examination [10]. Participants were recruited from population-based listing from four regions in the United States (Baltimore, MD, Pittsburgh, PA, Minneapolis, MN, and Portland, OR) [10]. Women with a history of bilateral hip replacement or who were unable to walk without the assistance of another person were excluded from participation. Black women were also excluded in the original enrollment for SOF given a low incidence of hip fracture in this demographic. Subsequently, at the Year 10 SOF examination conducted between 1997 and 1998, 662 AfricanAmerican women were enrolled in the study increasing the total cohort enrollment to 10,366 women [11].

Of the original 9704 white women, 7008 (96\% of active participants) completed at least the questionnaire component of a Year 10 examination (between 1997 and 1998). Of those 2696 women who did not participate, 2081 had died before the visit and 290 had terminated study participation. All 662 African-American women completed this examination, bringing the total enrollment at the Year 10 examination to 7670 . Of the 7670,5470 completed an in-clinic examination of which 5346 provided serum specimens. Of the 5346, a subset of 1302 was randomly selected for renal function measurement. Of these, 1289 women with measurements of both SCr and Scysc at the time of the Year 10 exam and data available regarding vital status through April 2012 comprised the analytical cohort for this study. Thus, the observation period for this study spans the time of the Year 10 exam (1997-1998) with follow-up for the primary outcome of death through April 2012 (mean follow-up for mortality $9 \pm 4$ years).

All women provided written informed consent, and the study was approved by the Institutional Review Board at each site.

2.2. Estimation of Renal Function. Serum from fasting morning blood collected at the Year 10 examination was processed and stored at $-70^{\circ} \mathrm{C}$ until being thawed. SCr and Scysc levels were measured on these previously frozen serum samples. Scysc and SCr assays were performed at the University of Minnesota Medical Center in 2010. Scysc concentrations were determined using a BN100 nephelometer (Dade Behring Inc., Deerfield, IL) using a particle-enhanced immunonephelometric assay (assay range $0.23-8.00 \mathrm{mg} / \mathrm{L}$ with interassay coefficient of variation (CV) of $4.0 \%$ at a level of $0.71 \mathrm{mg} / \mathrm{L}$ and $3.1 \%$ at a level of $1.75 \mathrm{mg} / \mathrm{L}$ [mean interassay CV 3.7\%]) and then converted to standardized values traceable to a certified reference material. SCr was measured with a Modular P Chemistry Analyzer (Roche Diagnostics, Indianapolis IN) using an enzymatic method equation calibrated with materials traceable to an isotope-dilution mass spectrometry (IDMS) reference measurement procedure. Interassay $\mathrm{CV}$ is $4.0 \%$.

We estimated GFR using the BIS1, CKD-EPI ${ }_{c r}$, BIS2, and CKD-EPI $I_{\text {cr,cysc }}$, and equations are shown in Appendix Table 1 in Supplementary Material available online at https:// doi.org/10.1155/2017/8216878.

2.3. Mortality. Women were contacted every 4 months after the Year 10 examination until May 2012 to ascertain vital status (mean follow-up $9 \pm 4$ years); $95 \%$ of these contacts were completed in surviving participants. Cause of death was confirmed by death certificate and, when available, hospital discharge summaries. We used ICD-9 codes to classify causes of death as CVD (codes 401-405, 410-415, 425, 427.5, 428, 429.2, 430-439, 440-445, and 798).

2.4. Other Measurements. Demographic data such as race and education level were taken from baseline examination for white women and Year 10 examination for Black women. Body-mass index (BMI, $\mathrm{kg} / \mathrm{m}^{2}$ ) as a measure of obesity, smoking status, and self-reported health status were obtained at the Year 10 examination. Comorbid conditions as defined by self-report alone at the Year 10 examination included stroke, coronary heart disease (CHD), and congestive heart failure (CHF). Hypertension (HTN) and diabetes mellitus (DM) were defined as self-report or use of antihypertensive or antihyperglycemic medications, respectively.

2.5. Statistical Analysis. We used the Chi-square test to compare the prevalence of CKD in 4 categories of eGFR $\left(>75,60-74,45-59\right.$, and $<45 \mathrm{~mL} / \mathrm{min} / 1.73 \mathrm{~m}^{2}$; henceforth 
TABLE 1: Prevalence of eGFR category using 4 different estimating equations.

\begin{tabular}{lcccc}
\hline \multirow{2}{*}{ GGFR category, $\mathrm{mL} / \mathrm{min} / 1.73 \mathrm{~m}^{2}$} & \multicolumn{3}{c}{ GFR estimating equation, $n(\%)$} \\
& BIS1 & CKD-EPI $_{\text {cr }}$ & BIS2 & CKD-EPI \\
\hline 75 & $159(12.3)$ & $622(48.3)$ & $432(14.1)$ & $432(33.5)$ \\
60 to $<75$ & $495(38.4)$ & $358(27.8)$ & $485(37.6)$ & $430(33.4)$ \\
45 to $<60$ & $481(37.3)$ & $209(16.2)$ & $124(9.6)$ & $284(22.0)$ \\
$<45$ & $154(12.0)$ & $100(7.8)$ & $143(11.1)$ \\
\hline
\end{tabular}

eGFR, estimated glomerular filtration rate; BIS, Berlin Initiative Study; CKD-EPI, Chronic Kidney Disease Epidemiology Collaboration; cr, creatinine; cysc, cystatin C.

eGFR units are removed for ease of reading) between each eGFR equation. We compared baseline characteristics of participants included in this analysis across 4 categories of eGFR using ANOVA and $\chi^{2}$ test for continuous and categorical variables, respectively.

We then determined age-adjusted all-cause and CVD mortality rate (deaths/1000 person-years) during the followup time by category of baseline eGFR for each estimating equation. We calculated unadjusted hazard ratios for death by eGFR category using Cox proportional hazards regression. We further adjusted for factors determined a priori to be clinically relevant potential confounders of the association between renal function and death (age, race, BMI, DM, and HTN) and other factors that were associated with both predictor and outcomes. Thus, we present the following models: (1) an unadjusted model (site only); (2) a base model, further adjusted for age, race, and BMI; (3) a multivariate model, further adjusted for HTN and DM; and (4) a final model, further adjusted for CHD and self-reported health status.

Finally, we calculated a metric comparable to the net reclassification improvement (NRI) for each formula compared to the CKD-EPI ${ }_{\text {cr,cysc }}$ equation (see Appendix Table 2 for NRI formulae) [12]. The point estimate of the NRI is interpreted as follows: a positive NRI, total for the estimating equation evaluated means that the equation more often appropriately reclassifies to a HIGHER eGFR (better function) those participants who lived and/or to a LOWER eGFR (worse function) those who died when compared to the referent equation $\left(\mathrm{CKD}-\mathrm{EPI}_{\mathrm{cr}, \mathrm{cysc}}\right)$. We also present a category-free NRI which defines reclassification as 1 unit change in eGFR that allows us to provide estimates that may be more comparable across studies that may not choose the eGFR categories we have used in our analyses [13].

In secondary analyses, we repeated all of the above analyses expressing eGFR in quartiles as the outcome (see Appendix Tables 3 and 4). All significance levels reported were two-sided and all analyses were conducted using SAS 9.4 (SAS Institute Inc., Cary, NC).

\section{Results}

3.1. Prevalence by eGFR Categories and Baseline Characteristics. The mean age of women in this cohort was $79.5 \pm 4.6$ years and $90 \%$ were white. Mean BMI was $27 \pm 5 \mathrm{~kg} / \mathrm{m}^{2}$. Mean follow-up time was $9 \pm 4$ years. The BIS2 equation identified $47 \%$ and $10 \%$ as having eGFR $<60$ and eGFR $<45$, respectively, compared to $33 \%$ and $11 \%$ by the $\mathrm{CKD}-\mathrm{EPI}_{\mathrm{cr}, \mathrm{cysc}}$ equation. The
BIS1 equation identified $49 \%$ and $12 \%$ as having eGFR $<60$ and eGFR $<45$, respectively, compared to $24 \%$ and $8 \%$ for the CKD-EPI cr $_{\text {equation }}(p<0.001$ for all pairwise comparisons, Table 1).

Women in a lower eGFR category, regardless of formula used, were older, more likely to be white, with poorer health status, and more likely to have a history of hypertension, stroke, CHD and CHF. In addition, women with lower CKD-EPI ${ }_{c r, c y s c}$ and BIS2 eGFR had higher BMI. Worse renal function was associated with greater prevalence of DM, though this trend was statistically significant only across BIS2 eGFR category. Characteristics of participants according to category of CKD-EPI ${ }_{\text {cr,cysc }}$ are displayed in Table 2.

3.2. Estimated GFR and Risk of Death. Over the follow-up period, 667 (51.8\%) women died overall and 239 (18.3\%) of women experienced a CVD death. In unadjusted analyses, lower eGFR category (worse function) calculated using any one of the four equations was associated with a graded, increased risk of all-cause mortality that was similar in magnitude across eGFR formulae (Table 3). Women with eGFR $<45$ compared to those with eGFR $\geq 75$ had a 2.9 -fold higher risk of death by BIS1 eGFR and a 3.1-fold higher risk of death by CKD-EPI ${ }_{\text {cr }}$ eGFR ( $p$-trend across category of eGFR $<0.001$ for both equations). Women with eGFR $<45$ compared to those with eGFR $\geq 75$ had a 3.2-fold higher risk of death when eGFR was calculated using CKD-EPI ${ }_{c r, c y s c}$ formula and a 3.7-fold higher risk of death when eGFR was estimated using BIS2 equation ( $p$-trend across category of eGFR $<0.001$ for both equations). Further adjustment for age, race, BMI, comorbid conditions, and health status somewhat attenuated the point estimates of the associations, though point estimates of the association were similar between each BIS equation and its respective CKD-EPI comparator formula (HR [95\% CI] for eGFR <45 versus $\geq 75$ : BIS1, 1.5 [1.1-2.0]; CKD-EPI ${ }_{\mathrm{cr}}, 1.7$ [1.3-2.2]; BIS2, 2.0 [1.4-2.8]; CKD-EPI ${ }_{c r, c y s c}, 1.8$ [1.4-2.3]). When we defined renal function by quartiles of eGFR, the point estimates were less robust, but similar significant trends across quartiles of eGFR were observed (Appendix Table 3).

When CVD mortality was substituted for all-cause mortality outcome in the analyses, lower eGFR category calculated using each one of the four equations was similarly associated with higher risk of CVD death across formulae (Table 4). Point estimates of the association of eGFR with CVD death in the final multivariable model were similar to those in the all-cause mortality analysis, despite borderline statistical significance. When renal function was expressed by 


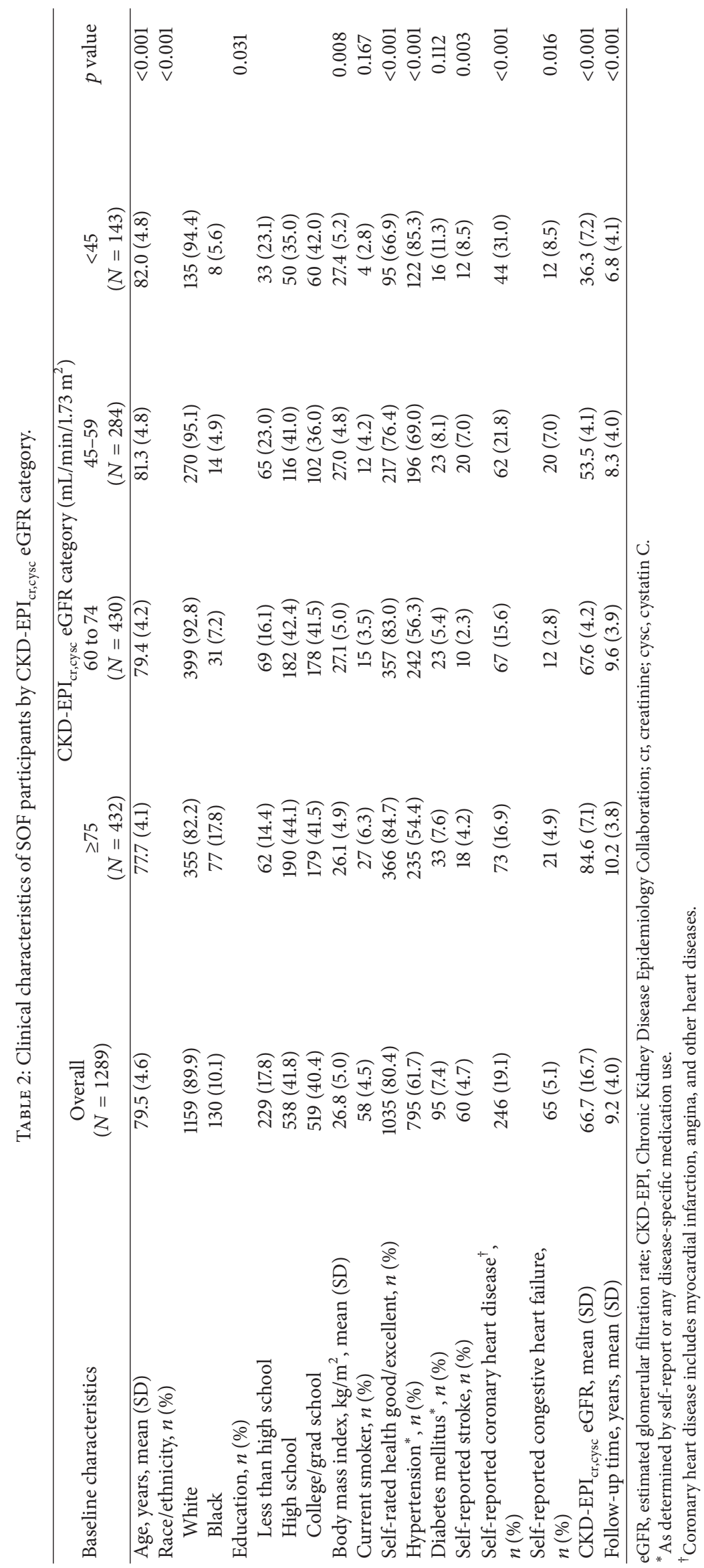




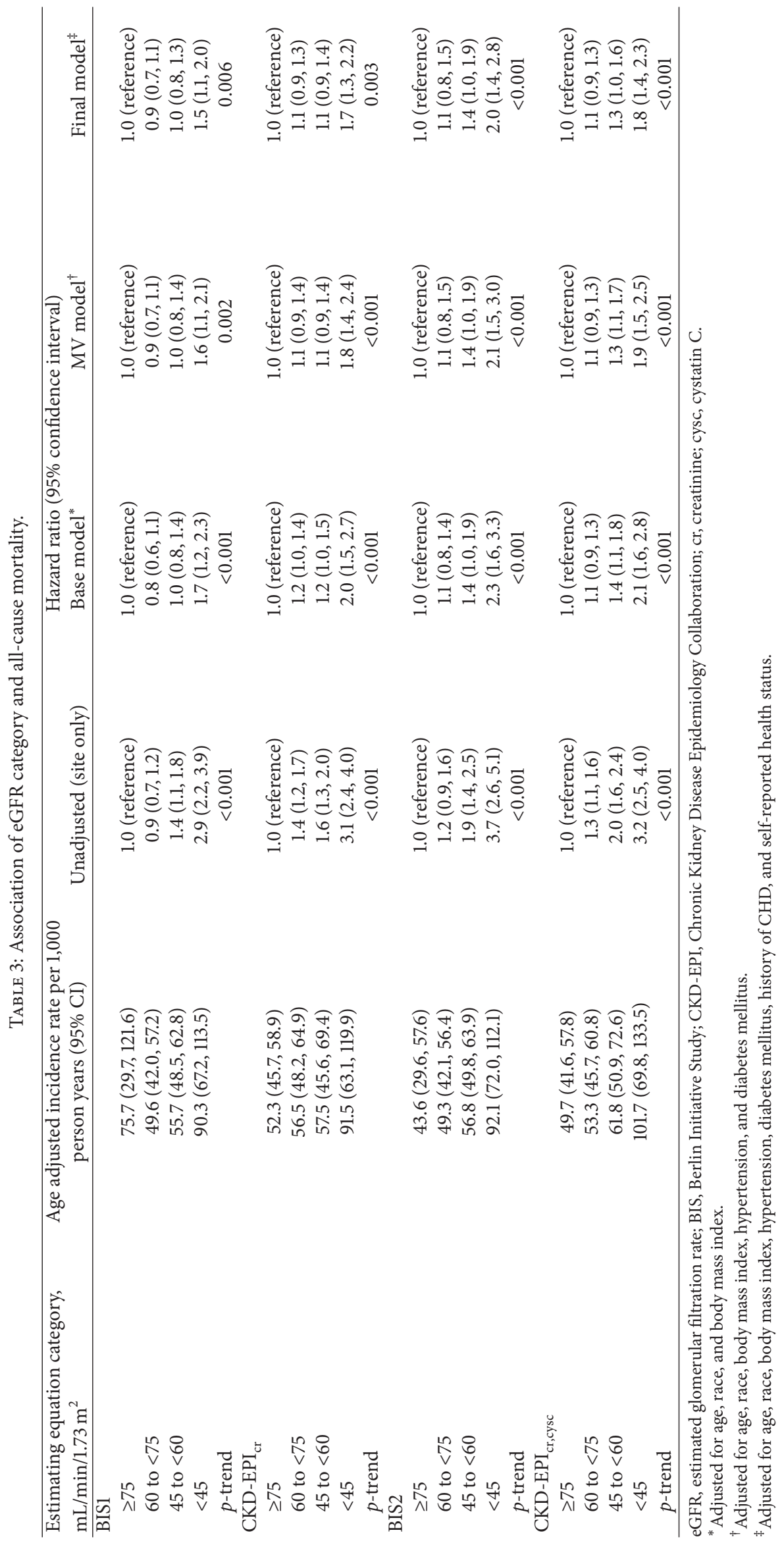




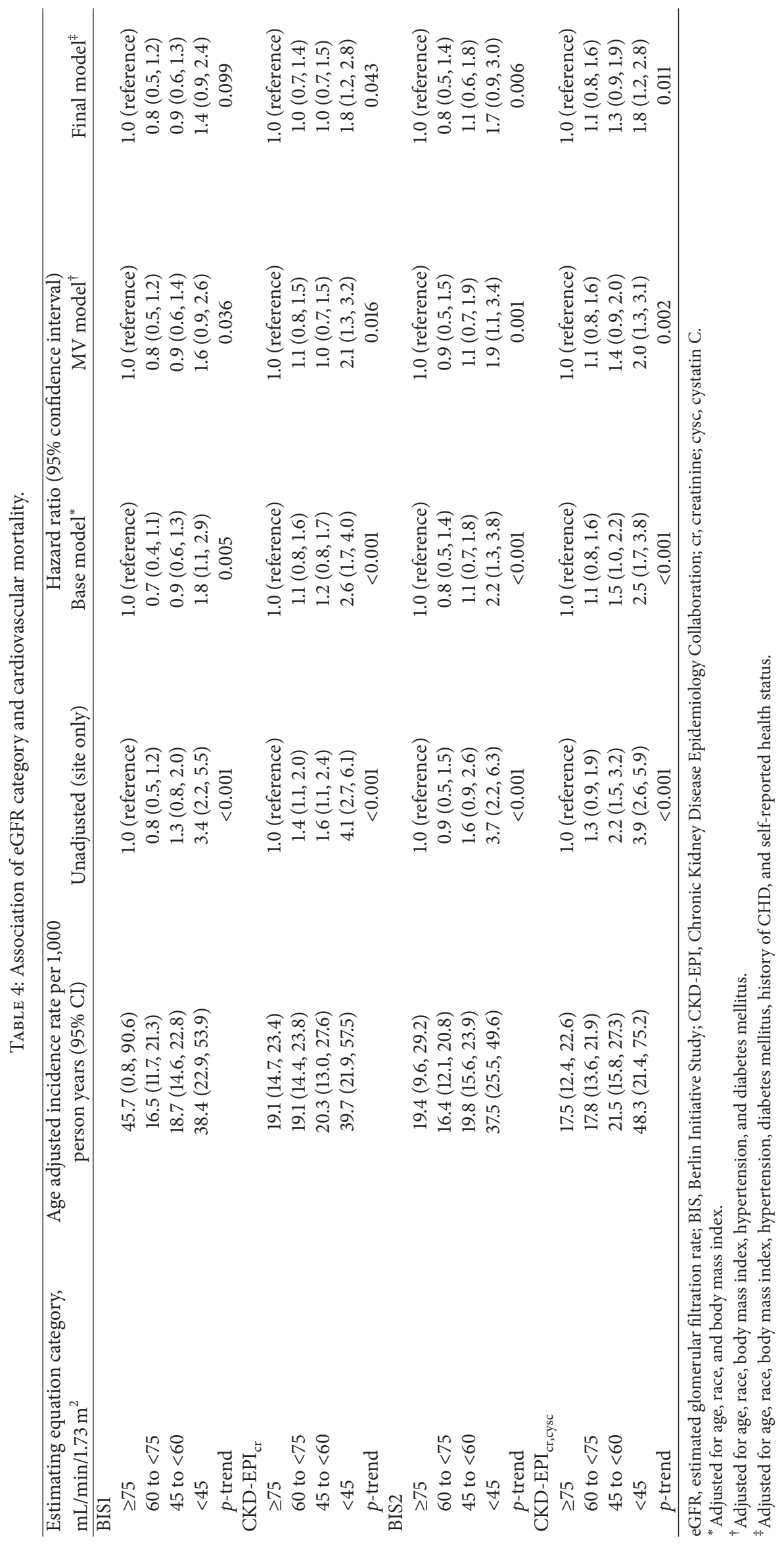


quartiles of eGFR in analyses with CVD death as the outcome variable, we observed similar trends across quartiles, though the point estimates were less robust than when we used eGFR categories and were not statistically significant after adjusting for age, race, and BMI (Appendix Table 4).

Finally, in category-based NRI analyses (Table 5), the BIS1 equation when compared to the CKD-EPI ${ }_{c r}$ equation more appropriately categorized women who died to a lower eGFR category. However, to a greater degree, the BIS1 inappropriately categorized women who did not die to a lower eGFR category. Overall, the net reclassification favored less appropriate risk classification for BIS1 compared to that for the CKD-EPI ${ }_{\mathrm{cr}}$, though this difference was not statistically significant. We observed similar results for the BIS2 compared to the CKD-EPI ${ }_{c r, c y s c}$. Findings were not altered when analyses were repeated using the category-free classification of eGFR. When CVD mortality was the outcome, we noted similar risk-reclassification patterns in both category-based and category-free NRI analyses, though the point estimate of NRI for nonevents was more strongly in the direction of inappropriate recategorization to a lower eGFR for those who did not experience a CVD death. None of the NRI values were statistically different from those of the referent CKD-EPI equation.

\section{Discussion}

In this cohort of community-dwelling older women, lower eGFR irrespective of estimating equation used was associated with a higher risk of all-cause mortality and CVD death. The BIS eGFR equations, which were developed specifically to estimate kidney function in older adults, did not outperform the KDIGO-recommended CKD-EPI equations to predict death including CVD mortality in this population. Thus, our findings lend no support to the replacement of the CKD-EPI equations with the BIS equations to predict death in older women.

We found that nearly half of our participants had an eGFR $<60$ by either BIS equation compared to $27 \%$ and $33 \%$ for the CKD-EPI ${ }_{c r}$ and CKD-EPI ${ }_{\text {cr,cysc }}$ equations. These results are consistent with those of other studies examining the prevalence of CKD by the BIS and CKD-EPI formulae in both older women and men $[9,14-16]$. The prevalence of eGFR by either BIS equation was similar to that of directly measured GFR in other studies of older adults similar in age to our cohort, though these studies were not limited to women $[9,16]$. In addition, in previous studies of older adults, the BIS equations consistently identified a higher prevalence of CKD compared to the CKD-EPI equations [14, 15, 17]. For example, in an external validation study of the BIS equations in 394 men and women of median age 80 years, Alshaer et al. found that the BIS equations identified a greater proportion of the population as having lower eGFR, while the CKD-EPI equations overestimated renal function when compared to measured GFR [17]. Taken together, our findings in concert with those of others confirm that the BIS equations tend to identify a higher proportion of older adults as having CKD.

Furthermore, findings from other studies of older men and women have been consistent with our observation that the BIS equations do not outperform the CKD-EPI equations in predicting all-cause and CVD mortality $[14,15,18,19]$. A population-based prospective study of Italian men and women over 85 years old found that after 5 years' follow-up time the BIS1 did not outperform other equations including the CKD-EPI ${ }_{\text {cr }}$ equation to predict mortality. Another population-based cohort study of 1017 German men and women (59\% female) aged 71-75 years observed that the BIS2 equation more inappropriately classified participants who did not die to a lower eGFR category when compared to the CKDEPI $_{\text {cr,cysc }}$ [18]. Finally, a study of community-dwelling older US men similarly found that the BIS2 equation compared to the CKD-EPI ${ }_{\text {cr,cysc }}$ formula more inappropriately reclassified men to lower eGFR who did not die. While none of these studies were performed solely in women, their findings corroborate our observation that the BIS equations do not outperform the CKD-EPI equations with respect to mortality risk prediction.

The clinical implication of our study is of importance to both clinicians and researchers. The 2012 KDIGO guideline recommends a paradigm shift from the clinical standard MDRD eGFR equation to the CKD-EPI equations to estimate GFR [7]. For populations in which SCr-based estimates may be less reliable (e.g., older age), Scysc-based CKD-EPI equations are recommended [7]. However, the guidelines note that should other novel equations arise that are more applicable to certain populations, consideration of use of those equations is reasonable [7]. Considering that several novel equations have been developed in the past 10 years, this leaves the practitioner and researcher in a quandary regarding the best equation to meet their needs. To that end, our study provides useful information regarding the performance of a new equation specifically developed for community-dwelling older adults compared with that of guideline-recommended equations. Based upon our findings, the BIS equations should not replace the CKD-EPI equations to predict death on older community-dwelling women.

Our study has several key strengths that set it apart from other studies. First, to our knowledge, we are the first study to test the performance of the BIS equations against the CKD-EPI equations to predict death in a cohort entirely composed of older women. Our cohort had long-term followup and accurate ascertainment of outcomes and covariate data. Furthermore, we present equations based not only on SCr but also on SCr and Scysc, both of which are traceable to the international reference standard. This allowed us to compare the predictive validity of each BIS equation against a comparable CKD-EPI equation. In addition, the incorporation of Scysc in older adults may be important given studies that have shown that Scysc-based estimates are more sensitive predictors of death in older adults [20].

We also note that our study has limitations to consider. We lack generalizability to male populations given our cohort is entirely female. However, we have previously published similar findings in a cohort consisting only of older men, thus corroborating that our findings are consistent in women and men. In addition, our cohort is largely Caucasian and though $10 \%$ are Black, there is limited racial or ethnic diversity otherwise. The BIS equations were developed in entirely 


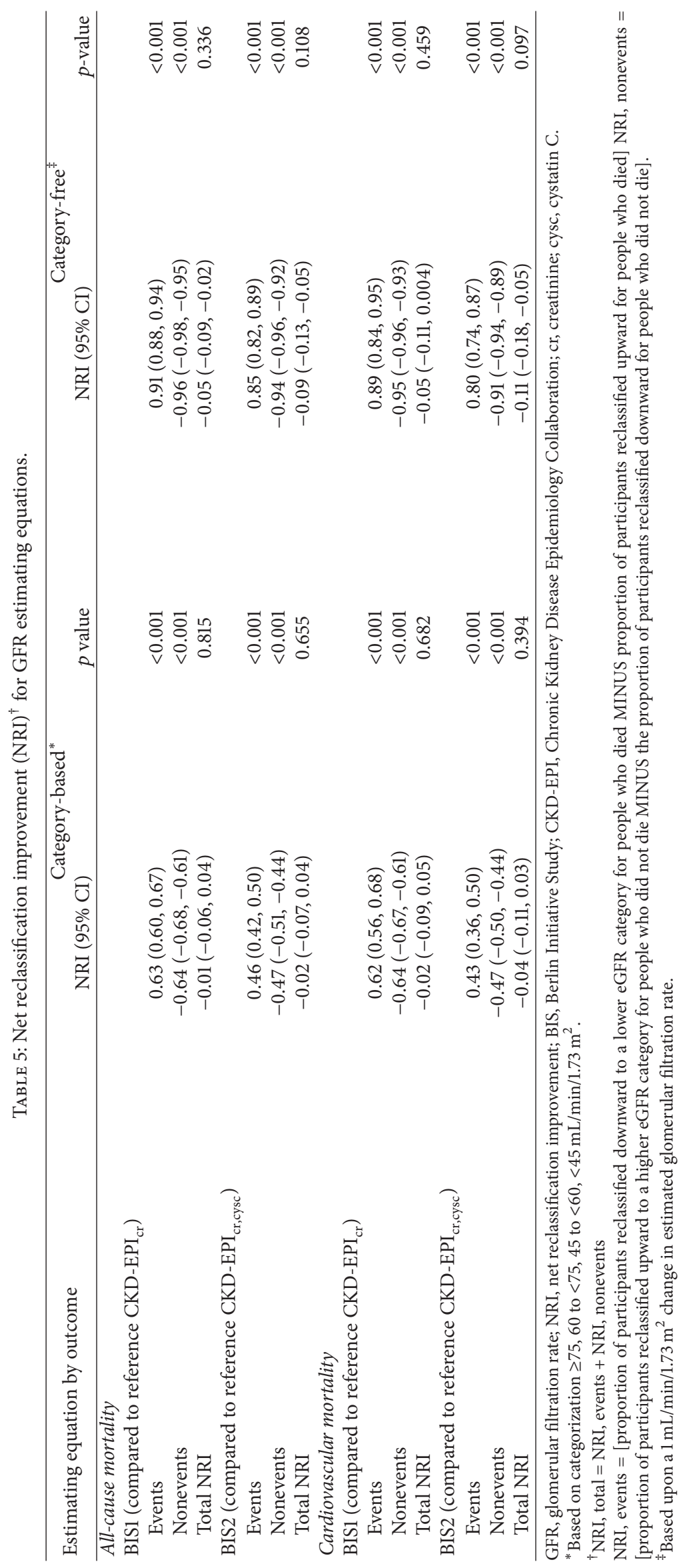


Caucasian population which makes SOF a fitting cohort in which to test the BIS equations. However, because of the limited diversity in the BIS Study, there is no race adjustment for those equations which may limit their reliability among Black SOF participants. Also, we lack information regarding a complementary marker of renal function and predictor of CKD-related death, albuminuria. However, because the purpose of this analysis was purely to compare the performance of 4 eGFR formulae to predict death, the addition of albuminuria across equations would not likely have changed our observations. In addition, we lack information on the cause of CKD, if present, or episodes of acute kidney injury during the follow-up period. These factors may be related to risk of CKD-related outcomes such as mortality. However, we have adjusted for common comorbid conditions that cause CKD such as DM and HTN. Finally, we cannot be certain that our estimates of renal function represent the true baseline eGFR of the participants because we do not have prior estimates of renal function in these population-based cohorts in which we measured SCr from stored serum from a single time point.

To sum, the BIS formulae identify a higher proportion of older community-dwelling women as having CKD. Our results suggest that the BIS equations do not outperform guideline-recommended CKD-EPI equations. Thus, with respect to predicting risk of death from estimated renal function in older women, our findings do not lend support for incorporation of the BIS equations into clinical practice or research.

\section{Conflicts of Interest}

The authors declare that they have no conflicts of interest.

\section{Acknowledgments}

The Study of Osteoporotic Fractures (SOF) is supported by National Institutes of Health funding. The National Institute on Aging (NIA) provides support under the following Grant no. R01 AG005407, R01 AR35582, R01 AR35583, R01 AR35584, R01 AG005394, R01 AG027574, and R01 AG027576. Dr. Canales's time is supported by a VA CSR\&D Career Development Award (CX000533-01A1) and by support from the Division of Nephrology, Hypertension and Renal Transplantation, Department of Medicine, University of Florida. This manuscript is also the result of work supported in part with resources and use of facilities at the Minneapolis VA Health Care System and the resources and facilities of the NF/SGVHS Medical Service, the Center of Innovation on Disability and Rehabilitation Research, and the Geriatric Research Education Clinical Center, Gainesville, FL. The contents herein do not represent the views of the U.S. Department of Veterans Affairs or the United States Government. The authors would also like to acknowledge Kyle Moen for his assistance in copyediting and formatting this manuscript. All authors are a subset of a larger research group (Study of Osteoporotic Fractures (SOF) Research Group) that designed the parent study.

\section{References}

[1] A. M. O’Hare, A. I. Choi, D. Bertenthal et al., "Age affects outcomes in chronic kidney disease," Journal of the American Society of Nephrology, vol. 18, no. 10, pp. 2758-2765, 2007.

[2] R. N. Foley, A. M. Murray, S. Li et al., "Chronic kidney disease and the risk for cardiovascular disease, renal replacement, and death in the United States medicare population, 1998 to 1999," Journal of the American Society of Nephrology, vol. 16, no. 2, pp. 489-495, 2005.

[3] D. S. Keith, G. A. Nichols, C. M. Gullion, J. B. Brown, and D. H. Smith, "Longitudinal follow-up and outcomes among a population with chronic kidney disease in a large managed care organization," Archives of Internal Medicine, vol. 164, no. 6, pp. 659663, 2004.

[4] A. S. Levey, L. A. Inker, and J. Coresh, "Chronic kidney disease in older people," Journal of the American Medical Association, vol. 314, no. 6, pp. 557-558, 2015.

[5] R. Glassock, P. Delanaye, and M. El Nahas, "An age-calibrated classification of chronic kidney disease," JAMA - Journal of the American Medical Association, vol. 314, no. 6, pp. 559-560, 2015.

[6] L. A. Inker, C. H. Schmid, H. Tighiouart et al., "Estimating glomerular filtration rate from serum creatinine and cystatin C," New England Journal of Medicine, vol. 367, no. 1, pp. 20-29, 2012.

[7] Kidney Disease: Improving Global Outcomes (KDIGO) and CKDWork Group, "KDIGO 2012 Clinical Practice Guideline for the evaluation and management of chronic kidney disease," Kidney International Supplement, vol. 3, no. 1, pp. 1-150, 2013.

[8] A. S. Levey, L. A. Stevens, C. H. Schmid et al., "A new equation to estimate glomerular filtration rate," Annals of Internal Medicine, vol. 150, no. 9, pp. 604-612, 2009.

[9] E. S. Schaeffner, N. Ebert, P. Delanaye et al., "Two novel equations to estimate kidney function in persons aged 70 years or older," Annals of Internal Medicine, vol. 157, no. 7, pp. 471-481, 2012.

[10] S. R. Cummings, M. C. Nevitt, W. S. Browner et al., "Risk factors for hip fracture in white women," New England Journal of Medicine, vol. 332, no. 12, pp. 767-773, 1995.

[11] M. T. Vogt, D. A. Rubin, L. Palermo et al., "Lumbar spine listhesis in older African American women," Spine Journal, vol. 3, no. 4, pp. 255-261, 2003.

[12] M. J. Pencina, S. D’Agostino, J. D’Agostino, and R. S. Vasan, "Evaluating the added predictive ability of a new marker: from area under the ROC curve to reclassification and beyond," Statistics in Medicine, vol. 27, no. 2, pp. 157-172, 2008.

[13] M. J. Pencina, S. D’Agostino, and E. W. Steyerberg, "Extensions of net reclassification improvement calculations to measure usefulness of new biomarkers," Statistics in Medicine, vol. 30, no. 1, pp. 11-21, 2011.

[14] G. Van Pottelbergh, B. Vaes, W. Adriaensen et al., "The glomerular filtration rate estimated by new and old equations as a predictor of important outcomes in elderly patients," $B M C$ Medicine, vol. 12, no. 1, article no. 27, 2014.

[15] S. Mandelli, E. Riva, M. Tettamanti, P. Detoma, A. Giacomin, and U. Lucca, "Mortality prediction in the oldest old with five different equations to estimate glomerular filtration rate: The Health and Anemia population-based study," PLOS ONE, vol. 10, no. 8, Article ID e0136039, 2015.

[16] L. Fan, A. S. Levey, V. Gudnason et al., "Comparing GFR estimating equations using cystatin $\mathrm{C}$ and creatinine in elderly individuals," Journal of the American Society of Nephrology, vol. 26, no. 8, pp. 1982-1989, 2015. 
[17] I. M. Alshaer, H. S. Kilbride, P. E. Stevens et al., "External validation of the berlin equations for estimation of GFR in the elderly," American Journal of Kidney Diseases, vol. 63, no. 5, pp. 862-865, 2014.

[18] I. E. Emrich, J. W. Pickering, B. Schöttker et al., "Comparison of the performance of 2 GFR estimating equations using creatinine and cystatin $\mathrm{C}$ to predict adverse outcomes in elderly individuals," American Journal of Kidney Diseases, vol. 65, no. 4, pp. 636-638, 2015.

[19] M. T. Canales, T. Blackwell, A. Ishani et al., "Estimated GFR and mortality in older men: are all eGFR formulae equal?" American Journal of Nephrology, vol. 43, no. 5, pp. 325-333, 2016.

[20] M. G. Shlipak, K. Matsushita, J. Arnlov et al., "Cystatin C versus creatinine in determining risk based on kidney function," New England Journal of Medicine, vol. 369, no. 10, pp. 932-943, 2013. 


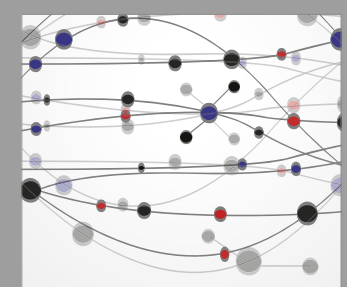

The Scientific World Journal
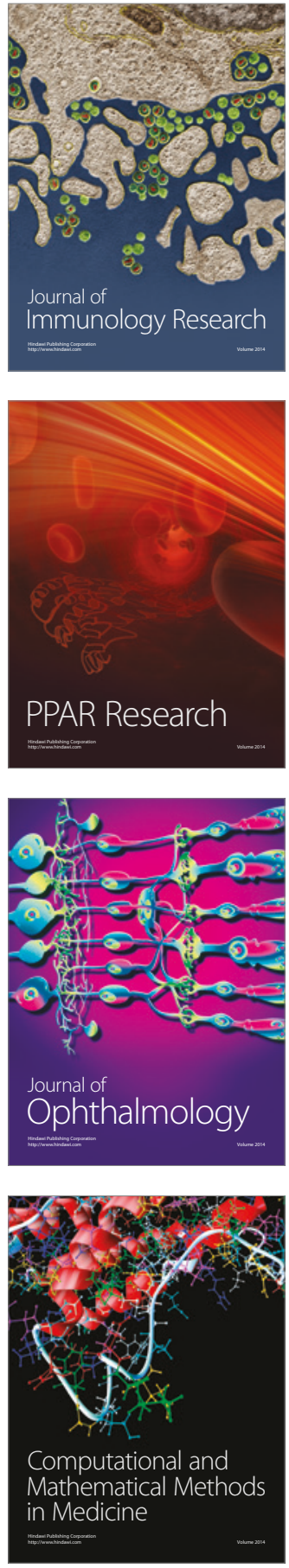

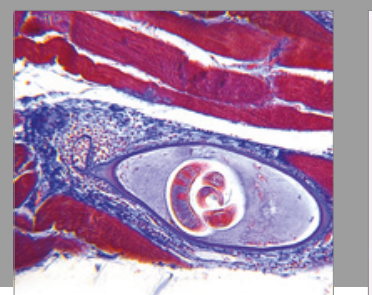

Gastroenterology Research and Practice
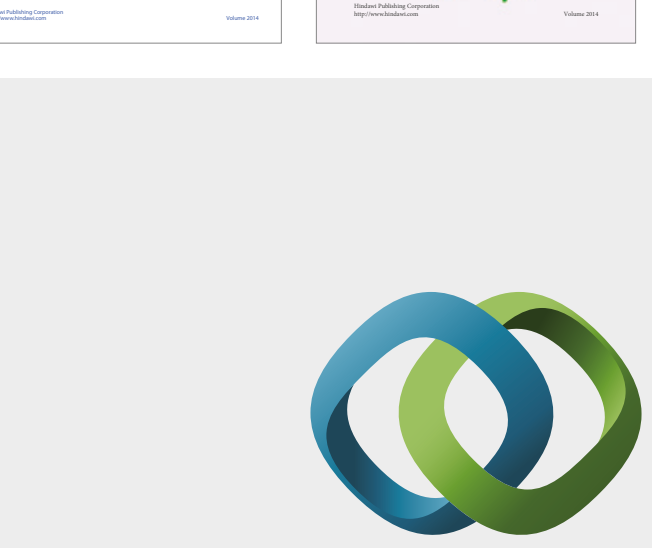

\section{Hindawi}

Submit your manuscripts at

https://www.hindawi.com
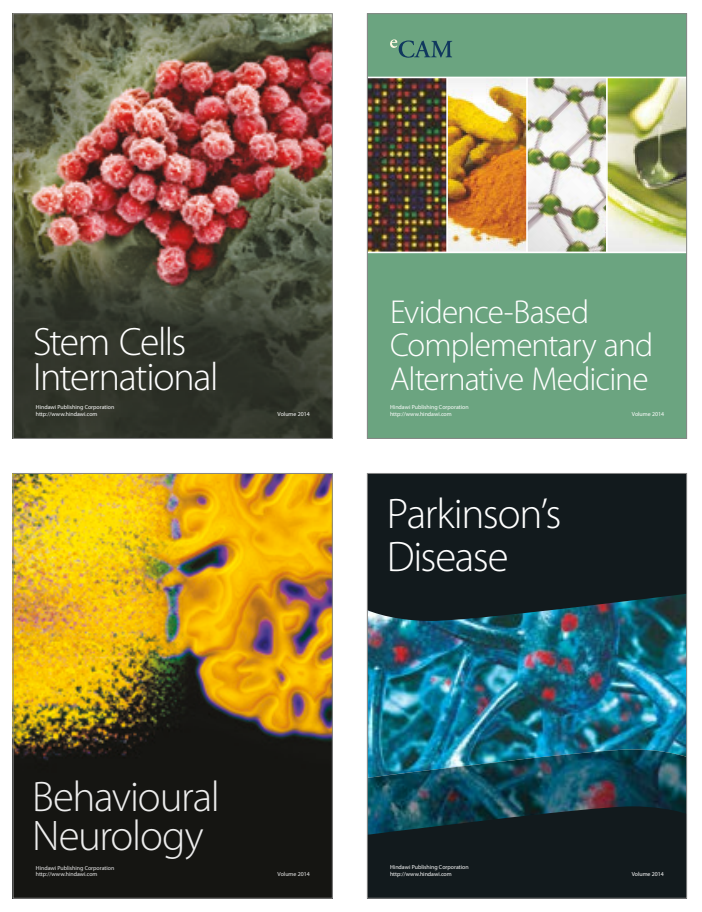
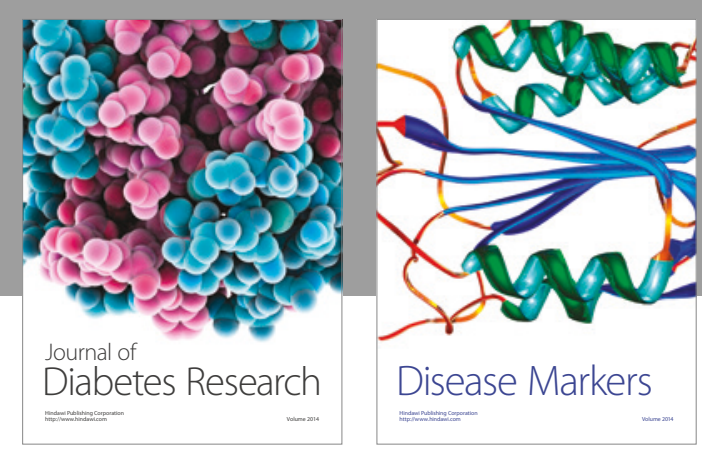

Disease Markers
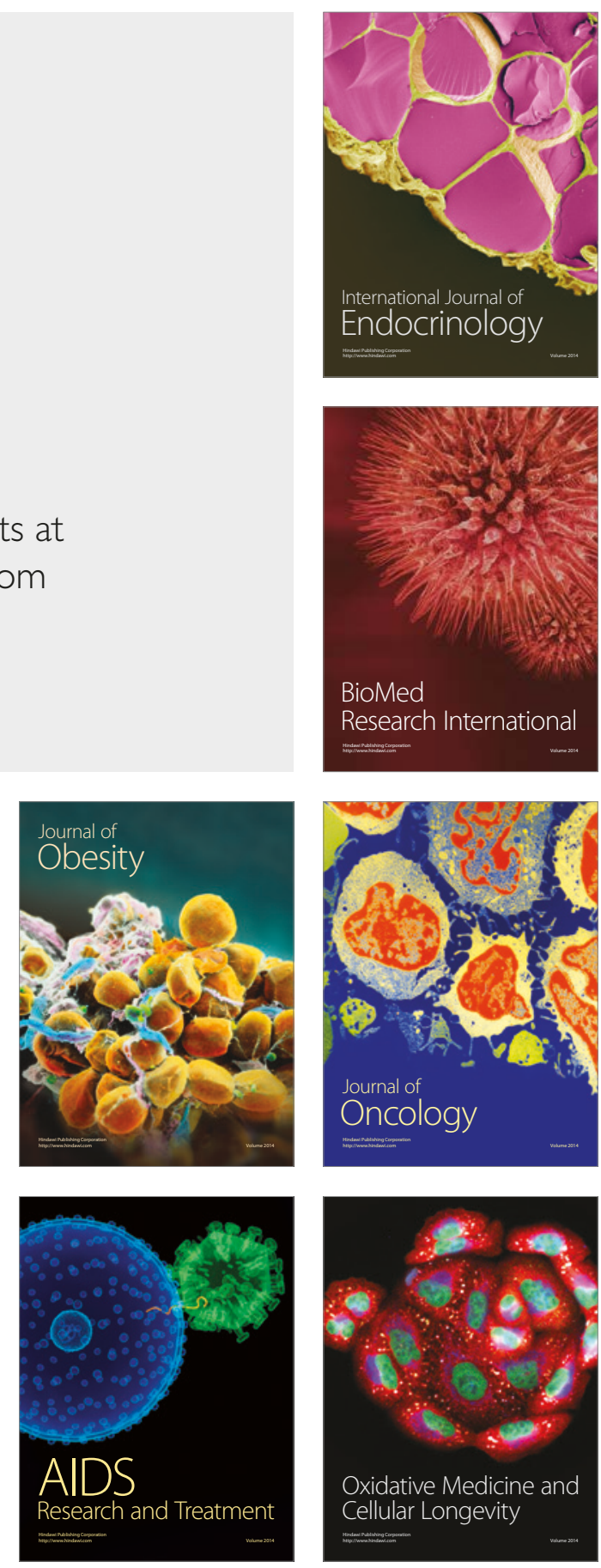